\title{
Agnès Gayraud, Dialectique de la pop
}

Paris, La Découverte, 2018

\section{Florent Lahache}

\section{(2) OpenEdition}

\section{Journals}

Édition électronique

URL : http://journals.openedition.org/transposition/5698

DOI : 10.4000/transposition.5698

ISSN : 2110-6134

Éditeur

CRAL - Centre de recherche sur les arts et le langage

\section{Référence électronique}

Florent Lahache, "Agnès Gayraud, Dialectique de la pop », Transposition [En ligne], 9 | 2021, mis en ligne le 15 décembre 2020, consulté le 23 avril 2021. URL : http://journals.openedition.org/transposition/ 5698 ; DOI : https://doi.org/10.4000/transposition.5698

Ce document a été généré automatiquement le 23 avril 2021

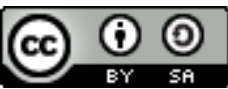

La revue Transposition est mise à disposition selon les termes de la Licence Creative Commons Attribution - Partage dans les Mêmes Conditions 4.0 International. 


\title{
Agnès Gayraud, Dialectique de la pop
}

\author{
Paris, La Découverte, 2018
}

\author{
Florent Lahache
}

\section{RÉFÉRENCE}

Agnès Gayraud, Dialectique de la pop, Paris, La Découverte, 2018, 528 p.

1 Un antagonisme embarrasse la théorie esthétique depuis maintenant un demi-siècle : celui qui oppose le grand art aux sous-cultures. Sitôt que cette opposition se formule, un scénario prévisible se met en route où l'une des branches de l'alternative sert à taper sur l'autre (élitisme bourgeois versus masse décérébrée). Pour fragiles qu'en soient les fondements, ce partage ne cesse pourtant de revenir, qu'il s'agisse de faire valoir la culture des dominés, celle d'un modernisme indépassable, ou bien d'enterrer leur conflictualité au sein d'un paradigme pluraliste. En pratique, la persistance de cette opposition est avant tout le symptôme d'une crise de la critique : au moment où le capitalisme et la culture en viennent à se superposer, où les hiérarchies artistiques s'effondrent et les industries du divertissement deviennent un lieu esthétique majeur, le partage qu'instituait la modernité entre l'art véritable et son double malfamé (la marchandise culturelle, le sous-produit kitsch, l'art de masse) paraît aussi binaire qu'inopérant. Cette situation oblige à repenser les principes d'une théorie de la culture qui abandonne l'idée de hiérarchie esthétique sans pour autant désactiver la fonction de la critique. La tâche n'est pas simple. Plutôt que reconstruire un grand modèle, une manière de s'y prendre consiste peut-être à prendre les objets un par un.

2 Cet enjeu est au centre de la Dialectique de la pop d'Agnès Gayraud, qui y propose une théorie de la musique pop, entendue non comme genre musical particulier (la Pop), mais comme paradigme général de la «musique populaire enregistrée » - soit, pour l'essentiel, le vaste domaine de la chanson enregistrée et diffusée mécaniquement. Le terrain d'exploration que dessine une telle définition est pour le moins considérable, des premiers field recordings aux grands labels contemporains, des anciens phonographes aux actuelles plateformes de streaming. Il est aussi profondément 
disparate, découvrant l'étendue des genres et sous-genres de la pop, folklorisants, excentriques ou insipides, des plus commerciaux aux plus clandestins. La manière dont la chanson s'est progressivement infiltrée dans la vie privée et partout ailleurs ( $\mathrm{du}$ supermarché au cinéma, du bistrot à la danse contemporaine) en fait un objet brûlant : elle constitue l'un des vecteurs les plus visibles du déplacement culturel qui s'est engagé depuis les années 1960.

3 Si l'ouvrage d'Agnès Gayraud, philosophe et musicienne, a reçu un très bon accueil, c'est qu'il répond en ce sens à une nécessité. Dans son développement, son propos nous paraît pourtant particulièrement peu convaincant. On essaiera ici de dire pourquoi, mais on commencera par dire qu'il ne s'agit pas d'un exercice facile. D'abord parce que Dialectique de la pop est un livre fort épais (516 pages), qui en contient plusieurs et dont chacun mériterait d'être discuté : un essai sur le statut de la pop comme industrie culturelle; une analyse serrée des positions de Theodor W. Adorno, traité comme archétype de la modernité anti-pop; une recension thématique des contenus discursifs des chansons; une réflexion sur les "hits » et les modalités du succès musical, ou encore sur la progressivité historique du matériau pop. Difficile, d'autre part, pour des raisons de méthode: le propos du livre n'est pas toujours cohérent d'un chapitre à l'autre et il est délicat d'en extraire une thèse qui ne serait pas contredite ailleurs dans le livre. C'est là la conséquence d'une étrange construction qui, au nom de la dialectique, s'autorise à énoncer des choses contradictoires plutôt qu'à mettre au travail des contradictions ${ }^{1}$, laissant régulièrement l'impression d'effectuer des repentirs, comme si le livre hésitait, cherchait sa mire, sa focale - comme s'il tournait un peu autour du pot.

4 Le pot, en l'occurrence, réside dans un genre de défense et illustration de la chanson, fondée sur une esthétique tendanciellement « universelle » ou « démocratique ${ }^{2}$ ». Pour en déplier l'argument, Agnès Gayraud développe trois propositions qui constituent le noyau théorique de son livre et que l'on pourrait reconstituer à peu près comme suit :

5 a) il existe une unité de la "musique populaire enregistrée » fondée sur son dispositif technique (l'enregistrement), sur les pratiques sociales et les modes de circulation (virtuellement indéfinis) qu'il rend possibles. Cette conceptualisation minimale dessine un objet à part entière, indépendant des autres pratiques de la musique (écrite, improvisée, concrète, etc.), en sorte qu'on peut l'examiner pour lui-même sans s'encombrer d'analyses musicologiques désobligeantes - nul besoin de comparer Madonna à Schönberg, ils ne font pas la même chose.

b) Cette musique laisse entendre des subjectivités ou des mondes sociaux ordinairement exclus de la représentation esthétique. La pop est la musique de n'importe qui adressée à tous, le medium de ceux que la virtuosité ou la technicité de la musique savante disqualifie par avance. Loin d'en avérer la dimension répétitive ou prévisible, la multiplicité même de la pop révèle au contraire des individualités singulières, exploite les petites déviances ordinaires, s'appuie sur l'originalité possible du quelconque.

7 c) Le charme de la pop repose sur une expressivité "naïve", "spontanée » ou " innocente », laquelle se trouve cependant captée par les médiations techniques et marchandes des industries culturelles, mais dont la chanson sait se rendre consciente. C'est dans cette tension que s'installe une « dialectique de la pop » : pour mettre en jeu son expressivité spécifique, la pop a besoin de médiations qui vont immanquablement 
l'aliéner ou la compromettre : tel est son drame ; en avoir conscience et savoir en jouer, tel est son génie propre.

8 Cette dialectique de la pop pourra paraître monotone ou indifférenciée (elle semble valoir indistinctement pour toute chanson), et ses catégories élémentaires (la naïveté et la réflexivité) pour le moins questionnables, mais la teneur de ces trois propositions charpente la part la plus consistante de l'ouvrage. La difficulté est ailleurs. Elle est dans ce qui vient parasiter ce geste même de détourage théorique, à savoir l'exercice de légitimation esthétique que s'impose le livre. Celui-ci s'accroche en effet à un problème inaugural - la déconsidération des industries musicales dans la doxa moderniste - mais qui paraît désormais bien inactuel. C'est comme registre désormais accepté sinon culturellement dominant que la pop est aujourd'hui à interroger. Dans une culture où n'existe plus ni canon ni avant-garde, et aucun principe ordonnateur du bon goût (où l'idée même de bon goût ne fait plus sens), la question de la légitimité semble sans objet. L'auteure en convient d'emblée, «la pop n'a plus guère d'ennemis » (p. 16). La persistance d'une illégitimité supposée tout au long de l'ouvrage fait donc énigme. Si la pop peut être décrite comme une pratique à part entière, c'est à elle-même qu'il faut la confronter et non à l'idéal esthétique d'avant-gardes depuis belle lurette évanouies. De ne pas tirer cette conséquence résulte un étrange quichottisme, où il s'agit de défendre la musique pop contre des adversaires imaginaires ou ressuscités, au choix.

Ce glissement a dans le livre un nom métonymique : Theodor W. Adorno, le théoricien des industries culturelles et contempteur de la musique légère. Le parti pris étant de dégager l'objet théorique " pop " à partir de ses adversaires, il s'agira d'en élire un, venu du passé mais valant pour tous - ainsi Adorno, érigé en modèle de « la rage » antipop (p. 34). Partant, le livre alterne entre lecture attentive du théoricien allemand ${ }^{3}$ et des moments de palpables exaspérations où les positions de ce dernier sont extrapolées afin de mieux les condamner. Ainsi, par exemple: «pendant des décennies, [le modernisme] a maintenu sa légitimité d'avant-garde, au moyen d'une culpabilisation autoritaire dont Adorno est en partie responsable. [...] L'esthétique adornienne a forgé une série de tabous : tabou de la légèreté, tabou de la tonalité, tabou du corps, tabou du mélange, tabou du lyrisme » (p. 136). Dans une version plus ramassée : «chez Adorno, la Modernité est entièrement dressée contre les musiques populaires, destinée à les exclure du royaume de l'art» (p.376). Ce genre d'assertions n'a guère de sens : on rappellera seulement que le concept d'industries culturelles qu'Adorno élabore dans les années 1940 avait précisément pour fonction d'informer la décomposition de la culture populaire ; dans la modernité industrielle, cette dernière ne perdure pour lui qu'à l'état de simulacre. Elle n'est ni un "enfer " ni un "paradis », elle est simplement absentée par la forme-marchandise. Accuser Adorno de vouloir la disqualifier, c'est feindre d'ignorer la place tragiquement fantomatique qu'elle occupe pour lui.

Nonobstant, Dialectique de la pop dépeint ainsi Adorno en grand inquisiteur moraliste. Le portrait qui en ressort est celui d'un théoricien irascible dont les motivations nous sont à peu près incompréhensibles (sur le contexte historico-politique dans lequel écrit Adorno, rien). À en suivre les pages, son projet n'était pas d'élaborer une théorie critique de la culture, mais de poser des interdits et de culpabiliser. La conséquence s'en déduit : l'enjeu du livre sera de déculpabiliser (on se demande tout de même qui) en théorisant une sorte de licence - à la "naïveté ", à la «simplicité», à "l'immédiateté ». La censure qu'il s'agit de lever est explicitement celle de la conscience critique (ou de la mauvaise conscience, ce qui revient ici au même) ${ }^{4}$. 
Curieuse manière de plaider la pop comme abandon de soi dans l'idéologie, quand on pourrait inverser le geste et tenter de repenser le concept même d'idéologie à partir de la pop. Il est révélateur sur ce plan qu'un terme disparaisse au fil des pages : celui de marchandise, et avec lui le questionnement touchant la fonction des œuvres dans le mode de production capitaliste, pourtant au cœur des analyses adorniennes.

Dialectique de la pop s'évertue ainsi à montrer qu'Adorno se trompe - la belle affaire. Au lieu de diviser sa pensée et de la rapporter à une histoire qui en particuliserait le jugement, il s'agit au contraire de l'absolutiser et du même coup d'en faire le porte-voix du projet musical moderne dans son ensemble. Il y a ici un chaînage où Adorno vaut pour l'art savant (réflexif, élitiste, «inécoutable»), qui vaut pour la modernité en général. Aussi influente qu'ait pu être la théorie adornienne, elle ne se confond pourtant pas avec la pensée et la pratique des compositeurs modernes. L'opération a ici quelque chose de stratégique : pour appuyer l'éloge de la pop, il s'agit d'instaurer Adorno en homme de paille de la modernité musicale.

Ou plus exactement de ce que le contemporain conçoit rétrospectivement comme ayant été cette modernité ${ }^{5}$. Le livre postule en effet une étrange entité historique, «la modernité artistique ", unanime dans sa condamnation du populaire. À supposer même qu'une catégorie aussi générique soit éclairante, le problème est qu'on la trouvera tout sauf homogène. De Béla Bartók à John Cage, de Maurice Ravel et Dmitri Chostakovich à Kurt Weill et Hanns Eisler, il n'est pas difficile de voir qu'une part essentielle de la modernité s'est emparée de l'impureté supposée du populaire et a construit des ponts entre la musique écrite et son dehors. Ce qu'il s'agit de défendre à travers cette construction, sans que ce ne soit jamais formulé, c'est manifestement le bien-fondé de la mélodie contre ceux qui ont voulu expérimenter autre chose. Voulant réfuter «le “tribunal esthétique" adornien » (p. 478), c'est bien une sorte de contre-tribunal qu'on voit se dresser au passage.

La simplification historique conduit à une logique de blocs: réflexivité versus spontanéité, modernité musicale versus pop - on n'en sort décidément pas ${ }^{6}$. Cette logique a un autre effet fatal, celui d'appréhender la pop elle-même de manière indifférenciée et dans des termes qui font problème. Le livre peine ainsi à proposer un modèle d'analyse de son objet : le traitement discursif et thématique des chansons y est majoritairement littéral (on apprendra ainsi que l'enfance est « une source intarissable de nostalgie » et que la vieillesse permet d'évoquer «l'expérience accumulée, parfois la sagesse » (p. 241) - le livre s'enfonce alors dans les lieux communs). On se demande également depuis quel point d'énonciation la catégorie de "naïveté » se trouve articulée (Booba ne pense sans doute pas qu'il fait de la musique naïve). Alors même qu'il s'agit d'en faire un usage mélioratif, le terme a quelque chose d'immanquablement surplombant, comme si l'auteure reconduisait sur le présumé populaire un point de vue bourgeois, celui-là même qu'elle s'évertue à conjurer - comme si elle était en fin de compte prisonnière des catégories qu'elle entendait défaire. Dans le même registre, «l'utopie de la réconciliation » censée animer la pop (comprendre : la musique savante exclut, la pop rassemble) contient une résonance étrangement pastorale ${ }^{7}$, laquelle se heurte à l'expérience ordinairement clivante de la musique pop, qui est au moins autant le lieu du goût que du dégoût des autres. La pop semble alors être un objet idéel, circulant dans une société foncièrement abstraite: le différend qu'elle aurait la vertu d'y annuler n'est jamais nommé. Que cette réconciliation ne soit jamais qu'une "promesse" asymptotique renforce encore ce sentiment d'abstraction. Le recours 
répété tout au long du livre à cette idéalité de la « promesse » ressemble à un artifice de raisonnement : dire que la pop n'est pas un accomplissement mais sa promesse, c'est faire passer une impossibilité structurelle pour un gage de bonne intention. À ce compte-là, n'importe quoi contient une promesse utopique. En sorte que le concept de pop qui ressort du livre ressemble à un tour de passe-passe : reprendre le concept d'industries culturelles, moins la violence sociale du capitalisme.

$\mathrm{Au}$ fondement du livre, on trouve un problème bien réel : une approche unilatérale des industries culturelles est devenue impossible; il existe désormais un paradigme pop qui traverse l'ensemble de la culture, et celui-ci désarme la pensée critique. Mise en position tout à la fois d'autorité et de repoussoir, la pensée d'Adorno s'avère peu éclairante pour penser cette situation. Dès lors que la musique légère n'était pas réellement son objet ${ }^{8}$ et que le pop comme concept et paradigme s'est constitué largement après lui, le livre donne lieu à des raisonnements désajustés, au bord du quiproquo. La chanson a changé de statut, c'est un fait. Depuis quand? Pour quelles raisons? Quelle périodisation en proposer? Coincé par une approche ontologique quand son objet est éminemment historique, l'ouvrage cale à l'endroit de ses propres partis pris théoriques. Car l'enjeu est sans doute moins de défendre l'existence de la culture pop que de savoir comment l'analyser, c'est-à-dire d'interpréter ces étranges objets sociaux que sont les œuvres sous condition du capital. Sur ce plan, rien n'indique que la notion-tourniquet de "populaire» produise la moindre intelligibilité sur la situation.

\section{NOTES}

1. Ainsi par exemple : «tout le monde aime bien la pop »(p. 16), même si «l'affirmation de la pop comme art dépend de ceux qui refusent de l'écouter » (p. 22). Le populaire contient «un projet utopique» (p. 78), même s'il peut «se renverser en repoussoir cauchemardesque » (p. 80), jusqu'à devenir un « chantage » autoritaire (p. 144). Dans un cas comme dans l'autre, il manque le plan de montage de ces revirements: une fois posé ce "même si ", l'auteure passe à autre chose. Plus largement: alors que le livre tout entier cherche la spécificité de la pop par opposition à la musique savante, il conclut sur l'évidence que le savant et le populaire «se rencontrent et se dialectisent à l'intérieur des pratiques musicales pop elles-mêmes» (p. 479). On se demande alors quel était l'enjeu du livre. On pourrait également mentionner la notion de «modernité artistique ", qui semble ne pas avoir la même acception dans la première et la seconde moitié du livre, renvoyant tantôt au grand art en général, tantôt aux avant-gardes formalistes en particulier, brouillant ainsi le plan de lecture.

2. «La musique populaire l'est au sens politique et esthétique. Le populaire n'y désigne point exclusivement une origine, il est une destination. Il promet en l'art l'universel accès: l'utopie d'une immédiateté qui ne serait pas trompeuse mais gage même de vérité, d'une démocratie esthétique sans aucun nivellement » (p. 481). 
3. Voir par exemple les analyses concernant la «lucidité » des consommateurs des industries culturelles, p. 112 et sq.

4. " $\mathrm{Au} \mathrm{xx}^{\mathrm{e}}$ siècle, chansons, films de genre, bandes dessinées ont assemblé des communautés esthétiques, formulé des rêves cosmiques, recyclé et inventé des mythes que les arts anciens, privés d'un droit à la naïveté par l'état avancé de leur conscience, de leur réflexivité, ne pouvaient plus imaginer sans se juger régressifs ou ridicules. » (p. 83).

5. Sur ce point, voir notamment l'ouvrage de CROW Thomas, Modern Art in the Common Culture, New Haven (Connecticut), Yale University Press, 1996, qui montre comment le postmodernisme a reconstruit une conception de l'art moderne afin de mieux pouvoir le condamner.

6. Même si finalement (il faudra quelques 400 pages pour y venir) la pop s'avère ne pas être si étrangère à la logique esthétique de la modernité - et donc conquérir à son tour toute sa légitimité.

7. «La langue pop - et la plus parfaite - se rapproche du parler en langues des Apôtres de la Bible : lorsqu'ils s'exprimèrent à Jérusalem devant une foule d'hommes de toutes origines (...), chacun les comprit pourtant comme s'ils s'adressaient à lui dans sa langue maternelle » (p. 315).

8. Sans surprise, les critiques les plus intéressantes qu'Agnès Gayraud adresse à Adorno concernent la musique savante elle-même (voir par exemple, p. 400 et sq.).

\section{AUTEURS}

\section{FLORENT LAHACHE}

Florent Lahache est professeur de philosophie à l'École des beaux arts de Bordeaux. Ses recherches portent sur les problématiques liées à la notion de culture postmoderne et l'actualité de la Théorie critique. Il a traduit (avec Marlène Monteiro) le livre de Laura Mulvey, Au-delà du plaisir visuel (Paris, Mimésis, 2017), et celui de Fredric Jameson, Brecht et la Méthode (Paris, Trentetrois morceaux, 2020). Il est par ailleurs éditeur pour les éditions Amsterdam. 